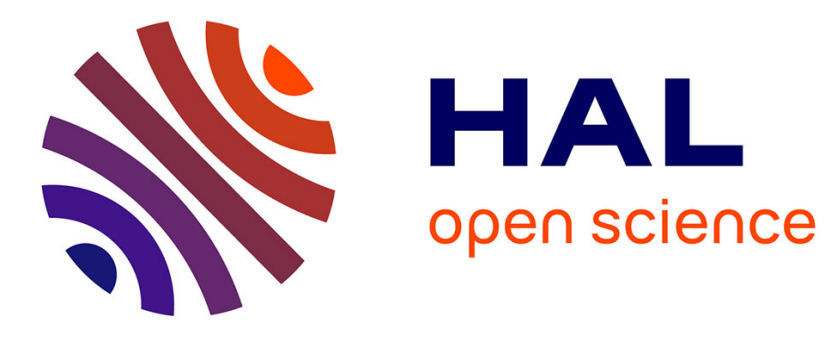

\title{
Circus Noel: A Case Study into Interaction and Interface Design for Cinematic VR
}

Mirjam Vosmeer, Alyea Sandovar

\section{To cite this version:}

Mirjam Vosmeer, Alyea Sandovar. Circus Noel: A Case Study into Interaction and Interface Design for Cinematic VR. 17th International Conference on Entertainment Computing (ICEC), Sep 2018, Poznan, Poland. pp.223-227, 10.1007/978-3-319-99426-0_21 . hal-02128621

\section{HAL Id: hal-02128621 \\ https://hal.inria.fr/hal-02128621}

Submitted on 14 May 2019

HAL is a multi-disciplinary open access archive for the deposit and dissemination of scientific research documents, whether they are published or not. The documents may come from teaching and research institutions in France or abroad, or from public or private research centers.
L'archive ouverte pluridisciplinaire HAL, est destinée au dépôt et à la diffusion de documents scientifiques de niveau recherche, publiés ou non, émanant des établissements d'enseignement et de recherche français ou étrangers, des laboratoires publics ou privés. 


\title{
Circus Noel: A case study on natural user interface design for VR
}

\author{
Mirjam Vosmeer ${ }^{1}$, Alyea Sandovar ${ }^{2}$, \\ ${ }^{1}$ Lectorate Play and Civic Media ${ }^{2}$, Amsterdam University of Applied Sciences, \\ Amsterdam, Netherlands \\ m.s. vosmeerehva.nl \\ alyeadtinthue.com
}

\begin{abstract}
.
In this paper, we present a case study for Circus Noel, a tight rope walking act in which the use of movement in VR was explored. We utilized Ryan's immersion dimensions and Norman's guide for natural interfaces to test the immersion of feet movement in VR. Seven users were interviewed on the dimensions of immersion; their responses were coded using the Ryan's dimensions. The results of this case study show that the spatial dimension exists automatically in VR. Our findings also show that the 'illusion of non-mediation' may be a useful concept to further explore the ways to measure, evaluate and validate VR.
\end{abstract}

\section{Introduction}

Immersive VR, we mean by this VR that is recorded with a $360^{\circ}$ camera also known as surround video or cinematic VR, provides the user with a new way to experience interactive entertainment in a cinematic setting. Users may experience a feeling of being surrounded by the movie that they are watching. Also users may feel connected to the story or the characters within the scene in a way that differs from engaging with a traditional movie, television show or video game. However, creating this kind of media entertainment also poses new challenges. For example, producers are confronted with a medium for which design conventions such as interface design and interaction design are still being developed. The possibilities for interface and interaction in VR need to be better understood to create experiences that fully explore the possibilities offered by VR. Alongside moviemakers and multimedia producers, researchers from different fields of academic expertise are exploring the questions that VR raises from a theoretical point of view. They seek insights into issues regarding, for instance, guiding the user's attention in a certain direction (Nielsen et al, 2016; Lin et al, 2017), or interactive storytelling structures (Kratky, 2016).

Outside of the field of cinema and academia, the attention for VR is growing exponentially as well. One of the factors for the growth of VR is the quick succession of VR devices that are being brought to the market, each version 
improving on usability. Due to its immersive qualities, VR is often considered a medium useful for different purposes, ranging from purely commercial and salesdriven opportunities to educational and therapeutic applications (Berntsen, 2016) However, the experimental and academic explorations into VR and the commercial expectations are diverging. But while VR companies are confronted with a steady flow of requests to create a variety of 'immersive' VR experiences, knowledge of how to produce high quality narrative VR content is developing at a slower pace. Another important issue that both producers and researchers are subsequently confronted with is the method with which VR experiences can be evaluated. As the field is still quite novel, validated instruments to measure the effectiveness of technological tools or creative strategies are still lacking.

A concept considered useful in the evaluation of VR is presence, the theoretical notion that is closely related to the term immersion. Presence has already been discussed extensively long before cinematic VR was developed. However, the kind of presence that cinematic VR offers seems to differ from presence as experienced in other media, such as movies or video games.

This paper presents a VR project named Circus Noel, in which the possibility of movement in cinematic VR was explored. After an exploration of the theoretical notions of presence and immersion the results will be presented of a qualitative study that has been conducted using Circus Noel. Through user interviews of Circus Noel, we aimed to collect further insights into the ways that walking could be integrated in the interaction design of a VR experience and whether walking enhanced the storytelling qualities of VR. We thus intend to explore how movement in VR could increase immersion.

\section{Theoretical background}

The current study is part of a research program at the Amsterdam University of Applied Sciences titled Storytelling for $360^{\circ}$ Media. Through this project we explore the new technological tools or creative strategies that VR producers use to tell their story to their audience (Vosmeer and Schouten, 2014; Vosmeer, Roth and Schouten, 2015; Vosmeer and Schouten, 2017; Vosmeer, Roth and Koenitz, 2017). An important measure that can be used to determine the usefulness or effectiveness of separate tools or strategies is presence.

The term presence is usually defined as the 'sense of being there' (Ryan, 2015). It is differentiated from immersion by defining immersion as an objective criterion which depends on hardware and software. Presence is subsequently defined as the psychological more subjective sense of being in the environment, and mainly 
influenced by the content of the mediated world. Immersion could be seen as a quality of the medium, in this case a VR movie, while presence is a characteristic of the user experience (Roth, 2016).

\subsection{Narrative Immersion}

Narrative immersion is described as the effect that the structure and content of a story has on the user. Ryan reflects on narrative immersion as an important aesthetic goal for interactive narratives (Ryan, 2015). She claims that this immersion can be divided into four dimensions: spatial, temporal, spatiotemporal and emotional. Spatial immersion refers to the effects that the setting, the world and the place and time of the story have on the viewer. Temporal immersion alludes to the structure of the plot, how the story is oriented (character, plot or place-driven), including more traditional aspects of storytelling. Spatio-temporal immersion refers to the narrative point of view and audience integration. Finally, emotional immersion refers to the feelings that the viewer experiences from the story. Many traditional media are linked to emotional presence (for instance being immersed in a book, or a movie) that builds up over time, but a VR experience immediately offers a sense of spatial presence.

In a recent research study, it was also asserted that the two main dimensions of immersion are technical and narrative (Elmezeny, Edenhofer, \& Wimmer, 2018). These categories can be connected to the Ryan's descriptions (2015): (a) The "technology-induced experience of being surrounded by data" and (b) "the narrative experience of being imaginatively captivated by a story world". From this division, we propose that the first category may be influenced by body centered interaction (Slater \& Usoh, 1994; Slater, Usoh \& Steed, 1995), while the second is more connected to narrative transportation (Phillips \& McQuarrie, 2010).

\subsection{Evaluating Presence}

Lombard and Ditton (1997) have described presence as a 'perceptual illusion of non-mediation', indicating that in an ideal experience of presence, the user is no longer aware that there is technology involved. In this context, the term "technology" could refer to the pages of a book, the screen of a television or a head mounted display. With sensory induced presence, this illusion of non- 
mediation may be caused by technology that is working flawlessly, without bugs or lags or other cues that might distract the user's attention.

Vosmeer, Roth and Schouten (2015) used a standardized scale to measure presence in an investigation on the use of feedback in interactive VR. In this survey, presence was measured using the following items:

- I was a part of the environment in the presentation.

- I was actually there in the environment of the presentation.

- I was physically present in the environment of the presentation.

This study seemed to indicate that the items that were used indeed measured the concept of presence. In another study, however, use of the same scale did not show significant difference in presence between two groups of users (Vosmeer, Roth and Koenitz, 2017). One of the explanations that the authors offered for this result, is that the scale used for measuring presence was developed for interactive narratives but probably not fully sufficient to evaluate presence in VR. The current study therefore also aims to gather insights into what presence in VR actually feels like, in order to eventually formulate the right survey items to evaluate it.

\subsection{VR Interface design}

In order to not disrupt the immersive experience that VR offers and thus optimize the 'illusion of non-mediation', interface design for VR should be as non-intrusive as possible. For instance, the display of non-diegetic information within the VR scene or feedback about interaction should be avoided (Vosmeer, Roth and Schouten, 2015).

In their analysis of VR interaction paradigms, Pakkanen et al (2017) had their participants compare three different ways in which the user could interact within a $360^{\circ}$ video including hand gesturing, head orientation pointing and remote control use. It was found that the use of hand gestures was significantly more problematic than the other two types of interaction. This result possibly reflects that hand gestures which are 'new' to the users, are therefore more difficult to learn and to remember than the use of a remote control or head pointing.

A challenge with VR interface design is understanding which gesture yields a particular action for a user (Norman, 2010). Also, users require specific feedback on how to perform these gestures. They need to learn for instance how often and with how many fingers a movement should be executed. The use of 
these gestures should ideally feel as natural as possible for a user. Norman uses the example of graphical interfaces, stating that the strength of a graphical user interface has little to do with its use of graphics, but rather with the ease with which the required actions can be remembered. Consequently, interface design for VR should also focus on how much effort a user needs to interact with it and 'internalize' it.

\subsection{Movement in VR}

In his paper with the same title Gillies (2014) poses this question: What is Movement Interaction in Virtual Reality for? He proposes Slater's (2009) theory of Place Illusion, in which Slater states that the immersive quality of VR depends on the ability of the interactive design to reproduce the same sensorimotor contingencies as the real world. If a user experiences a VR system that supports many real world sensorimotor contingencies, the user is more likely to experience a form of presence called Place Illusion. A number of examples exist that show that movement interaction appears to increase the sense of presence in VR. Steinicke et al (2013) have stated that natural locomotion in VR would increase immersion, lessen simulator sickness and improve the user experience. In a study conducted in 1999, Usoh et al. found that walking in a digital environment created a stronger sense of presence than using a joystick.

There has also been some important criticism of movement based interaction. Norman (2010) has stated a number of issues regarding information systems lacking the capability to sense body language. One issue is that a person using a VR system is usually constrained to a limited set of actions that a computer can recognize and perform. In the case of walking in VR, the limitation may be the direction of movement. For instance, the user is only able to walk within the scope of the computers overview. It is the point of this paper to explore how movement in VR can be embedded in the interface design for this particular medium and thus increase immersion. In this context, walking can be redefined as using 'natural feet gestures'. The action of placing one foot in front of the other to cause a forward movement. This forward movement is an interaction design that does not require users to learn or internalize an unfamiliar gesture.

\section{Case study: Circus Noel}

Circus Noel was as a collaborative project between three students, an academic researcher, and two industry partners: a television broadcasting company 
(AVROTROS, 2018) and a VR production company (WeMakeVR, 2018). It is presented as a virtual tightrope walking act and was initially developed as a 'VR companion piece' alongside a television series for children that was broadcast on Dutch national in December 2017. Although the installation was built with a children's audience in mind, the VR experience was also successfully presented to an adult audience at Dutch festivals and demo sessions.

\subsection{The installation}

The goal of the project was to create a cinematic VR experience that allowed the user to physically walk through a VR scene. The experience is somewhat similar to other computer-generated VR experiences, such as The Walk by Sony (2016) or Richies's Plank Experience, by Toast (2016). The Circus Noel VR experience was filmed in an actual circus while the $360^{\circ}$ camera slide high through the air along a rope. Users can imagine themselves as a tightrope walker who is performing a circus act while fellow circus performers watch and cheer as the mission is accomplished. Producing a VR walking experience filmed with a $360^{\circ}$ camera, however, was a technical and conceptual challenge not often undertaken before.

To give the user a realistic sense of movement we build a physical installation that features a thin bar on the floor, between two platforms. Users wore a HTC Vive headset and HTC Vive trackers were attached to both ankles.

The experience begins with the user outside the tent where a circus assistant invites 'you' to step up and perform your tight rope walking act. She hands 'you' a pair of shoes, after which the viewer is transported onto a platform high in the circus tent. Looking down, the user can see the circus ring, in which other circus performers are present, and a rope that stretches towards the other side of the tent. The two shoes that were handed over before are seen below, and as the movements of her feet are monitored by the trackers, she can now imagine herself walking high in the air on the rope, while in reality she is walking on a thin bar, just a few centimeters above the ground. 


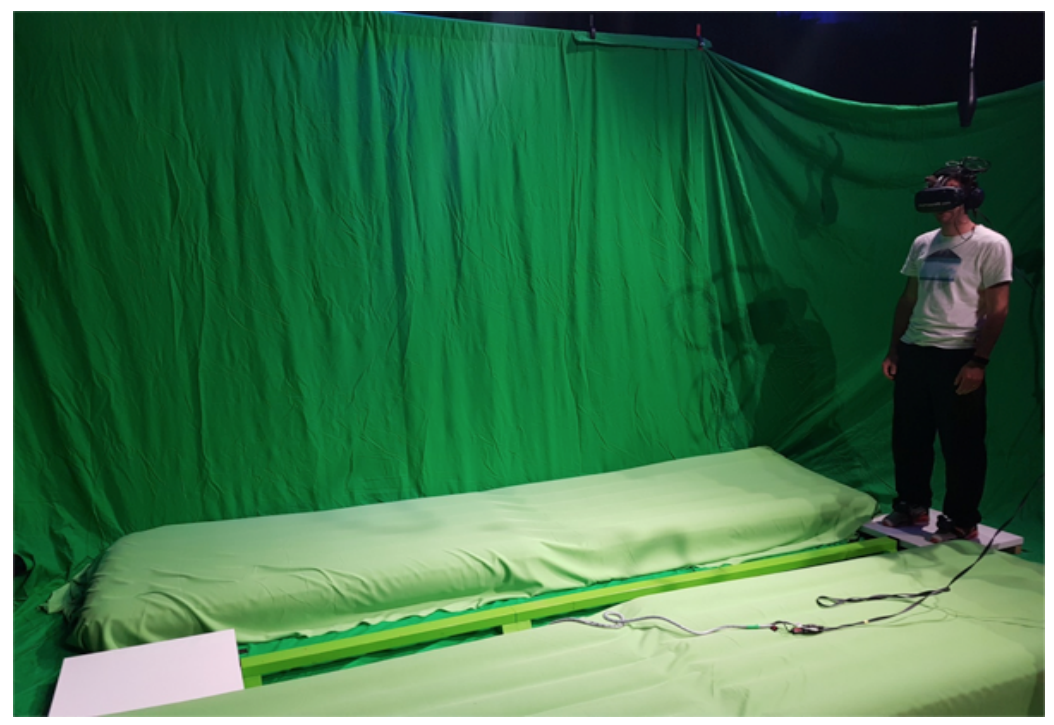

Fig. 1: Wearing a HTC Vive headset and trackers on both ankles, in reality the user balances on a thin bar between two platforms.

\subsection{Combining 3D and Film}

The circus setting within the VR experience mimics a real-life experience, but the tightrope as it is shown beneath the user, the two platforms that she walks between and her 'own' feet are 3D rendered. To accomplish the blending of the two realities, the filmed material was projected within a sphere using Unity, after which the rope, platform and shoes were added. 


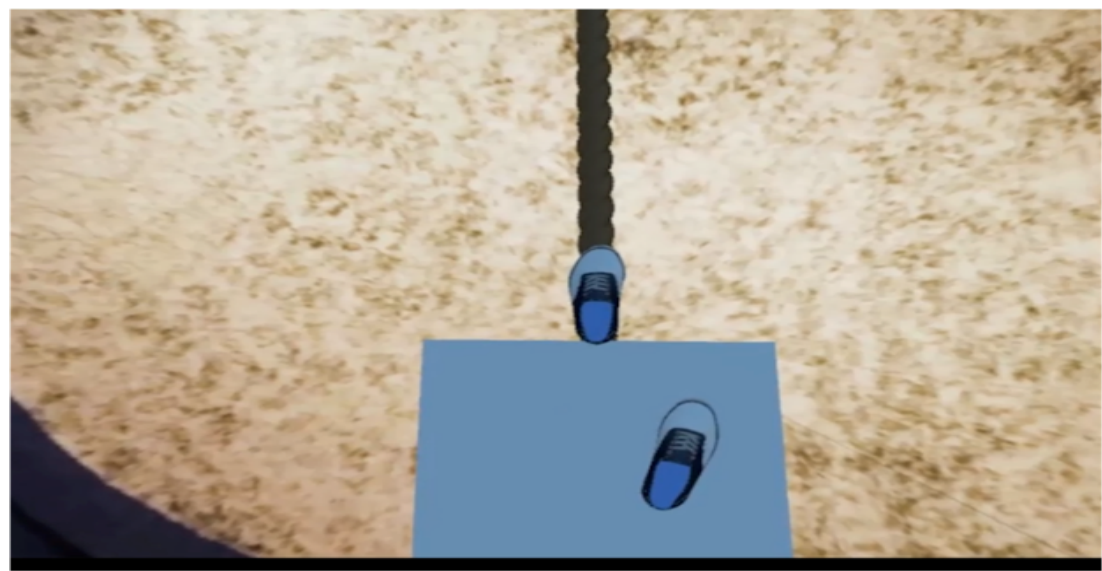

Fig. 2. The user sees her 'own' feet as she balances on the tightrope, high above the ground.

The footage was filmed using a Falcon VR camera. This particular model has 14 cameras on the side, and two cameras on the bottom and on the top. Especially the bottom cameras were important for our current project, as the circus ring down below should be perfectly visible.

To make the combination of the 3D generated rope and platform with the filmed images, these elements are placed in Unity. The filmed images are on a sphere projected in Unity, and the user is placed within this sphere. The 3D objects, the rope, the platforms and the shoes are put therein.

\section{The qualitative study}

The installation has been presented on several occasions to users of all ages. In one of these occasions users responded to an open-ended questionnaire about Circus Noel. The aim was to gather information on whether the user experience had felt real, how walking in VR felt to the user, and how the user had interpreted her own role:

- Was it you, who did the walking?

- Did the feet that you saw in VR feel like your own feet?

- Did the circus feel real or unreal? Why was that?

- Were you still aware of your surroundings here?

- Did you feel uncomfortable, being somewhere else? 
A total of 7 adults were interviewed about their experience, between the ages of 20 and 39 . Their responses were documented in a google survey.

\subsection{Results}

The answers to the open-ended questionnaire were coded and separated based on Ryan's (2015) levels of immersion: Temporal, spatial, spatial-temporal and emotional. The following summarizes some of the users' perspectives and immersive experience based on each of Ryan's dimensions.

The first dimension was the temporal dimension. When users were asked if they were aware of the story, they responded that indeed they knew they were replacing a tightrope walker that had quit his job because he had suffered from fear of heights. Some of the statements included: "They were looking for a tightrope act"; "It was some sort of audition"; "Yes, I knew who I was." Users did not see a body while walking, but rather just a pair of shoes that mimicked their steps as they walked on the plank. Interestingly, some even reported that having a whole body could alter the experience of immersion. One user responded: "I think a whole body could be distracting, because then you have to look at that as well, while focusing on the rope."

The spatial dimension was also explored in Circus Noel. When users were asked to describe their level of immersion in the environment, they all responded that they did feel they were in a tight rope experience. "I really felt like I was up there." "I thought it was scary and exciting. When I was walking the bar, I felt like I would fall down because it seemed really high." Some participants were only subtly aware of the people outside of the experience. One respondent shares "Consciously you know where you are. But it does not feel like that. It's strange to hear voices (of the researchers) when you can't see the people themselves." Sometimes users responded feeling silly for not wanting to look inadequate as they took the first step in the rope experience.

The difference between the virtual rope and the wooden plank that users needed to walk in the real world was sometimes a bit tricky. While users responded that the walking on the wooden plank mirrored the VR rope experience, it was not a 1 to 1 relationship. In addition, the $3 \mathrm{D}$ elements in the experience were not as realistic as the real world. The rope was a bit thicker, the art (shadowing) and other artistic expressions were not as realistic for the user. However, users seem to understand that it was "good enough" for an immersive experience, as one replied "I think it's really appropriate. It did not necessarily have to be realistic, but it worked well enough." 
The spatio-temporal dimension appeared to be quite successful. This perhaps is the dimension that most closely aligns to Norman's (2010) gesturing for creating a natural interface. When users were asked about their point of view and level of identification with their 'avatar feet', they responded that indeed the movement of their feet in the game and their real feet did correspond one to one "I felt that I really was walking. The blue bar is exactly as wide as the thing you feel. So it is good one on one." In terms of the timing and when users became aware of their feet, this spatio-temporal experience was also well immersed. The timing coincided with their personal movements and did not need to be adjusted. For example one person responded that she did not need to be oriented to her avatar prior to walking, being given the virtual shoes for the tightrope experience which "I did not really pay attention to her, only when she gave me the shoes. I was mostly looking around at the environment." Also, users did not feel weird or awkward with the trackers around their ankles.

When asked about their sense of balance, all users responded that it was harder to balance than usual. This indicates that the technical immersion, described by Ryan (2015) as "technology-induced experience of being surrounded by data" was indeed experienced by users. "Yes, I really felt that I would fall down if I would make a wrong step. Because it really seemed that I was high up in the air."

The emotional dimension appeared to have an interesting review. When users were asked if the experience itself was scary, some reported that indeed they had felt some anxiety mixed with slight feelings of uncomfortableness and at times excitement: "I found it scary and exciting. I had to walk across the bar. I felt like I would fall off because it seemed very high." Other users did not fear the height themselves, but stated that they could imagine it could be scary: "I can imagine that if you have fear of heights, then this is scary. I myself did not know how high the bar was in the real world. So I really did not want to fall down." This emotional immersion also reflects Ryan's "the narrative experience of being imaginatively captivated by a story world". Indeed, users experienced emotionally many of the effects that a new tight walker might experience. However, in a lessened state as these were in a safe environment. One user remarked "This experience helped me with addressing my fear of heights, knowing that I could experience this, changed my personal story about heights". 


\section{Conclusion and Discussion}

To start from the perspective of entertainment, walking in VR appears to offer an enjoyable and exciting interactive entertainment experience. On the whole, Circus Noel reflects the Ryan's dimensions for immersion (2015). In many cases, from the phrases that the users used to describe their rope-walking adventure, it can be deduced that they experienced an 'illusion of non-mediation'. For instance, if someone is referring to 'my feet', or is expressing a fear of falling, or is indeed feeling difficulty to keep their balance, this indicates that they are no longer quite aware of the medium itself, but instead are being grabbed by the "technologyinduced experience of being surrounded by data" (Ryan, 2015). This also becomes clear from the suggestions that some of the users added. When asked how the experience could be optimized further users had many suggestions. For example, users suggested that juggling could be added while balancing on the beam. Also suggested was increasing the level of difficulty by having flying objects cross over the beam. Another suggestion was to involve the audience by giving them virtual objects that they could control and through in the path of the trapeze so that several participants could experience the virtual experience at once. All this feedback indicates that the walking in VR experience did create a sense of immersion for the user, as they are in no way concerned with technical complications that their ideas would stand for, but instead speak solely from a perspective of entertainment. It can be concluded that the interface design that consists of creating movement by moving your feet - which can be seen as 'natural feet gestures' - instead of for instance having to use a controller or learn hand movements, indeed can help to optimize an immersive entertainment experience. Our further theoretical steps will include explorations on how the 'illusion of non-mediation' may be captured in survey items and set up a standardized questionnaire to measure, evaluate and validate movement inVR.

\section{References}

AVROTROS (2018). https://www.avrotros.nl

Berntsen, K., Palacios, R. C., \& Herranz, E. (2016, November). Virtual reality and its uses: a systematic literature review. In Proceedings of the Fourth International Conference on Technological Ecosystems for Enhancing Multiculturality(pp. 435-439). ACM.

Elmezeny, A., Edenhofer, N., \& Wimmer, J. (2018). Immersive Storytelling in 360-Degree Videos: An Analysis of Interplay Between Narrative and Technical Immersion. Journal For Virtual Worlds Research, 11(1).

Gillies, M., \& Kleinsmith, A. (2014). Non-representational interaction design. In Contemporary Sensorimotor Theory (pp. 201-208). Springer, Cham. 
Y.-C. Lin, Y.-J. Chang, H.-N. Hu, H.-T. Cheng, C.-W. Huang, and M. Sun (2017). Tell me where to look: Investigating ways for assisting focus in 360 video. In CHI, 2017

Lombard, M., \& Ditton, T. (1997). At the Heart of It All: The Concept of Presence. In Journal of computer-mediated communication. Pennsylvania: Temple University.

Kratky, A. (2016). Metaphor and Storytelling in Interface Design for Virtual Reality. In International Conference on Universal Access in Human-Computer

Interaction : $287-300$

Nielsen T., Møller, M. B., Hartmeyer, S. D., Ljung, T., Nilsson, N. C., Nordahl, R., \& Serafin, S. (2016, November). Missing the point: an exploration of how to guide users' attention during cinematic virtual reality. In Proceedings of the 22nd ACM Conference on Virtual Reality Software and Technology (pp. 229-232). ACM.

Norman, D. A. (2010). Natural user interfaces are not natural. interactions, 17(3), 6-10.

Pakkanen T, Hakulinen J, Jokel T, Rakkolainen I, Kangas J, Piippo P, Raisamo R, Salmimaa M. (2017, March). Interaction with WebVR $360^{\circ}$ video player: Comparing three interaction paradigms. InVirtual Reality (VR), 2017 IEEE 2017 Mar 18 (pp. 279-280). IEEE.

Phillips, B. J., \& McQuarrie, E. F. (2010). Narrative and persuasion in fashion advertising. Journal of Consumer Research, 37(3), 368-392.

Roth C (2016). Experiencing Interactive Storytelling. PhD thesis. http://dare.ubvu.vu.nl/handle/1871/53840 Retrieved June 2017.

Ryan M-L (2015) Narrative as virtual reality 2: Revisiting Immersion and Interactivity in Literature and Electronic Media. JHU Press.

Slater, M., \& Usoh, M. (1994). Body centred interaction in immersive virtual environments. Artificial life and virtual reality, 1(1994), 125-148.

Slater, M., Usoh, M., \& Steed, A. (1994). Depth of presence in virtual environments. Presence: Teleoperators \& Virtual Environments, 3(2), 130-144.

Slater, M. (2009). Place illusion and plausibility can lead to realistic behaviour in immersive virtual environments. Philosophical Transactions of the Royal Society B: Biological Sciences, 364(1535), 3549-3557.

Sony (2016). The Walk. http://www.sonypictures.com/movies/thewalk/vr/

Steinicke, F., Visell, Y., Campos, J., \& Lécuyer, A. (2013). Human walking in virtual environments (pp. 199-219). New York: Springer.

Toast (2016). https://toast.gg/plank/

Usoh, M., Arthur, K., Whitton, M. C., Bastos, R., Steed, A., Slater, M., \& Brooks Jr, F. P. (1999, July). Walking $>$ walking-in-place $>$ flying, in virtual environments. In Proceedings of the 26th annual conference on Computer graphics and interactive techniques (pp. 359-364). ACM Press/Addison-Wesley Publishing Co..

Vosmeer M, Schouten B. (2014) Interactive Cinema: Engagement and interaction. In: International Conference on Interactive Digital Storytelling 2014. Springer International Publishing, pp 140-147.

Vosmeer M, Roth C, Schouten B (2015) Interaction in surround video: the effect of auditory feedback on enjoyment. In: International Conference on Interactive Digital Storytelling 2015, Springer International Publishing, pp. 202-210.

Vosmeer, M., \& Schouten, B. (2017). Project Orpheus A research study into $360^{\circ}$ cinematic VR. Proceedings of the 2017 ACM International Conference on Interactive Experiences for TV and Online Video, 85-90.

Van der Spek, E. D. (2011). Experiments in serious game design a cognitive approach. SIKS Dissertation Series No. 2011-36.

WeMakeVR (2018). http://wemakevr.com/ 